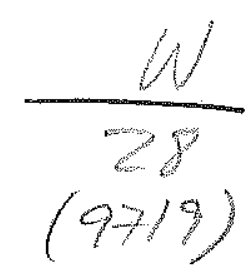

Documento de Trabajo 9719

INSURANCE CONSIDERING A NEW

STOCHASTIC MODEL FOR THE DISCOUNT FACTOR

Miguel A. Usabel

FACULTAD DE CIENCIAS ECONOMICAS Y EMPRESARIALES UNIVERSIDAD COMPLUTENSE DE MADRID VICEDECANATO

Campus de Somosaguas, 28223 MADRID. ESPAÑA. 
INSURANCE CONSIDERING A NEW STOCHASTIC MODEL FOR THE DISCOUNT FACTOR

MIGUEL A. USABEL 


\title{
Insurance considering a new stochastic model for the discount factor
}

\author{
Miguel USÁBEL \\ UNIVERSIDAd COMPLUTENSE DE MADRID
}

\begin{abstract}
In many empirical situations (e.g.:Libor), the rate of interest will remain fixed at a certain level(random instantaneous rate $\delta_{i}$ ) for a random period of time $\left(t_{i}\right)$ until a new random rate should be considered, $\delta_{i+1}$, that will remain for $t_{i+1}$, waiting time untill the next change in the rate of interest. Three models were developed using the approach cited above for random rate of interest and random waiting times between changes in the rate of interest. Using easy integral transforms (Laplace and Fourier) we will be able to calculate the moments of the probability function of the discount factor, $\mathrm{V}(\mathrm{t})$, and even its c.d.f.. The approach will also be extended to the calculation of the expected value(net premium) and variance of a term insurance and we will get its c.d.f., something not very common in actuarial literature due to its complexity, but very useful when the law of large numbers

cannot be applied and consequently use normal approximations.
\end{abstract}

\section{INTRODUCTION}

The stochastic instantaneous rate of interest has been frequently modelled using an Itô's process,

$$
d \delta(t)=\mu(\delta(t), t) d t+\sigma(\delta(t), t) d Z(t)
$$

where $\mu$ and $\sigma$ are the expected value and the variance, $Z(t)$ is a standard Wiener process. Many models were studied using this approach as stated in Hürlimann(1993), Ang and Sherris(1997) and Parker(1997). In these models, changes in the instantaneous rate of interest are implemented continuously.

In many empirical situations (e.g.:Libor), the rate of interest will remain fixed at a certain level(random instantaneous rate $\delta_{i}$ ) for a random period of time $\left(t_{i}\right)$ until a new random rate should be considered, $\delta_{i+1}$, that will remain $t_{i+1}$, waiting time until the next change in the rate of interest. This last fact means that a model with continuous changes in the instantaneous rate of interest may not be very appropriate.

Let us introduce the following approach. $V(t)$, the discount factor, can be defined

$$
V(t)=e^{-\left[\delta_{1} t_{1}+\delta_{2} t_{2}+\cdots+\delta_{n-1} t_{n-1}+\delta_{n}\left(t-\sum_{j=1}^{n-1} t_{j}\right)\right]}
$$

where: 
$-n$ is a random variable that represents the total number plus 1 of changes in the rate of interest within the time interval $(0, t]$ $-\left\{t_{j}\right\}_{j=1}^{n-1}$ the sequence on random variables that models the waiting times between changes in the rate of interest and $t_{j} \in(0, \infty)$ and $W_{j}(x)=P\left\{t_{j} \leq x\right\}$. It is clear that:

$$
\sum_{j=1}^{n-1} t_{j} \leq t \quad \text { if } n=1 \text { then } V(t)=e^{-\delta_{1} t_{1}}
$$

$-\left\{\delta_{j}\right\}_{j=1}^{n}$ is a sequence of random variables for the instantaneous rates of interest. Let us remember that $\delta_{j}=\ln \left(1+i_{j}\right)$ and assume that $\delta_{j} \in(0, \infty)$ and $F_{j}(y)=P\left\{\delta_{j} \leq y\right\}$.

Let us introduce the function:

$\gamma(t)=-\ln (V(t))=\left[\delta_{1} t_{1}+\delta_{2} t_{2}+\cdots+\delta_{n-1} t_{n-1}+\delta_{n}\left(t-\sum_{j=1}^{n-1} t_{j}\right)\right]$

then:

$$
\begin{gathered}
D_{t}(x)=P\{V(t) \leq x\}=P\left\{e^{-\gamma(t)} \leq x\right\}= \\
P\{-\gamma(t) \leq \ln (x)\}=P\{\gamma(t)>-\ln (x)\}
\end{gathered}
$$

if we now define:

$$
G_{t}(x)=P\{\gamma(t) \leq x\} \quad x \geq 0
$$

this expression is obtained:

$$
D_{t}(x)=P\{V(t) \leq x\}=1-G_{t}(-\ln (x))
$$

and $d_{t}(x)$ is the first derivative of $D_{t}(x)$.

Three models will be presented in section 2 using the approach cited above for random rate of interest and random waiting times between changes in the rate of interest. The first two (Models I.a and I.b) for independent rates of interest in the different waiting times and the last one for non-independent rates, more realistic in many situations (Model II).

In section 3, using Laplace and Fourier transforms, we will find, with Theorems 2 through 6 (based in renewal integral equations), expressions for the Laplace and Fourier transforms of a very interesting kind of functions directly related with $D_{t}(x)$.

In section 4, an elegant and direct expression for the moments of the probability distribution of the discount factor $\mathrm{V}(\mathrm{t})$ will be found for the three models considered. 
Later, in section 5 , we will see how the very c.d.f of $\mathrm{V}(\mathrm{t})$ can be obtained.

Finally, in section 6 , the promised extension to a term insurance is made and expressions for the net premium, variance and even the c.d.f. will be presented. Section 8 is devoted to numerical illustrations.

\section{FINANCIAL MODELS}

The following models for the rate of interest will be considered

\subsection{Model I.a.: i.i.d. rates of interest}

Let us assume that $\left\{\delta_{j}\right\}_{j=1}^{n}$ and $\left\{t_{j}\right\}_{j=1}^{n-1}$ are sequences(mutually independent) of i.i.d. random variables with common d.f. $f(y)$ and $w(x)$ respectively, then

$$
\begin{gathered}
\gamma_{I . a}(t)=\delta_{1} t_{1}+\delta_{2} t_{2}+\cdots+\delta_{n-1} t_{n-1}+\delta_{n}\left(t-\sum_{j=1}^{n-1} t_{j}\right) \\
G_{t}^{I . a}(x)=P\left\{\gamma^{I . a}(t) \leq x\right\} \quad x \geq 0
\end{gathered}
$$

with d.f. $g_{t}^{I . a}(x)$.

\subsection{Model I.b.: i.i.d. rates of interest with initial value.}

Let us consider now the case when $\delta_{1}=\delta_{1}^{*}$ is not a random variable and $t_{1}$ follows a d.f. $w_{1}(x)$ - that could be different from $w(x)$ - and the sequences $\left\{\delta_{j}\right\}_{j=2}^{n}$ and $\left\{t_{j}\right\}_{j=2}^{n-1}$ are formed with i.i.d. random variables with common d.f. $f(y)$ and $w(x)$ respectively, as it was stated in Model I.a.. The random variable $\gamma(t)$ could be written:

$$
\begin{gathered}
\gamma^{I . b}(t)=\delta_{1}^{*} t_{1}+\left(\delta_{2} t_{2}+\cdots+\delta_{n-1} t_{n-1}+\delta_{n}\left(\left(t-t_{1}\right)-\sum_{j=2}^{n-1} t_{j}\right)\right)= \\
\delta_{1}^{*} t_{1}+\gamma^{I . a}\left(t-t_{1}\right)
\end{gathered}
$$

if $t_{1}>t$ then $\gamma^{I . b}(t)=\delta_{1}^{*} t$.

Theorem 1. The c.d.f. of the random variable $\gamma^{I . b}(t)=-\ln (V(t)), G_{t}^{I . b}(x)$, can be expressed:

$$
G_{t}^{I . b}(x)=\frac{1}{\delta_{1}^{*} W_{1}\left(\frac{t}{\delta_{1}^{*}}\right)} \int_{0}^{\delta_{1}^{*} t} G_{t-\frac{\tau}{\delta_{1}^{*}}}^{I . a}(x-\tau) w_{1}\left(\frac{\tau}{\delta_{1}^{*}}\right) d \tau \quad x>0
$$


Proof. Let us define

$$
b_{1}=\delta_{1}^{*} t_{1} \Longrightarrow t_{1}=\frac{b_{1}}{\delta_{1}^{*}}
$$

with c.d.f. ,

$$
P\left\{b_{1} \leq x\right\}=P\left\{t_{1} \leq \frac{x}{\delta_{1}^{*}}\right\}=\frac{W_{1}\left(\frac{x}{\delta_{1}^{*}}\right)}{W_{1}\left(\frac{t}{\delta_{1}^{*}}\right)} \quad x \in\left(0, \delta_{1}^{*} t\right)
$$

then

$$
\gamma^{I . b}(t)=b_{1}+\gamma^{I . a}\left(t-t_{1}\right)
$$

if we consider $\left(\gamma^{I . a}\left(t-\frac{\tau}{\delta_{1}^{*}}\right) \mid \tau=b_{1}\right)$ is a conditional random variables with c.d.f. $G_{t-\frac{b_{1}}{\delta_{1}^{*}}}^{I . a}(x)$, bearing in mind that

$$
G_{y}^{I . a}(x)=0 \quad y \leq 0
$$

and using the total probability theorem

$$
\begin{aligned}
G_{t}^{I . b}(x)= & P\left\{\gamma^{I . b}(t) \leq x\right\}=\int_{0}^{\delta_{1}^{*} t} G_{t-\frac{\tau}{\delta_{1}^{*}}}^{I . a}(x-\tau) \frac{1}{\delta_{1}^{*}} \frac{w_{1}\left(\frac{\tau}{\delta_{1}^{*}}\right)}{W_{1}\left(\frac{t}{\delta_{1}^{*}}\right)} d \tau= \\
& \frac{1}{\delta_{1}^{*} W_{1}\left(\frac{t}{\delta_{1}^{*}}\right)} \int_{0}^{\delta_{1}^{*} t} G_{t-\frac{\tau}{\delta_{1}^{*}}}^{I . a}(x-\tau) w_{1}\left(\frac{\tau}{\delta_{1}^{*}}\right) d \tau
\end{aligned}
$$

for a fixed value of the initial instantaneous rate of interest. $\delta_{1}^{*}$.

\subsection{Model II: Non-independent rates of interest}

In this case we will assume that the sequence of random variables $\left\{\delta_{j}\right\}_{j=1}^{n}$ follow this pattern,

$$
\begin{aligned}
\delta_{1}= & \delta_{0}+\varepsilon_{1} \\
\delta_{2}= & \delta_{1}+\varepsilon_{2}=\delta_{0}+\varepsilon_{1}+\varepsilon_{2} \\
\delta_{3}= & \delta_{2}+\varepsilon_{3}=\delta_{0}+\varepsilon_{1}+\varepsilon_{2}+\varepsilon_{3} \\
& \vdots \\
\delta_{n}= & \delta_{n-1}+\varepsilon_{n}=\delta_{0}+\sum_{j=1}^{n} \varepsilon_{j}
\end{aligned}
$$

that means that the rates of interest are non-independent random variables. 
where $\delta_{0}$ is a fixed parameter and $\left\{\varepsilon_{j}\right\}_{j=1}^{n}$ are i.i.d. with common c.d.f.,

$$
P\{\varepsilon \leq x\}=H(x) \quad x \in(-\infty, \infty)
$$

random variable $\gamma(t)$ can be written, substituting 2.5 and operating

$$
\begin{aligned}
\gamma^{I I}(t) & =\delta_{1} t_{1}+\delta_{2} t_{2}+\cdots+\delta_{n-1} t_{n-1}+\delta_{n}\left(t-\sum_{j=1}^{n-1} t_{j}\right)= \\
& =\delta_{0} t+\varepsilon_{1} t+\varepsilon_{2}\left(t-t_{1}\right)+\cdots+\varepsilon_{n}\left(t-\sum_{j=1}^{n-1} t_{j}\right)
\end{aligned}
$$

\section{INTEGRAL TRANSFORMS OF $g_{t}(x)$ AND $\mathbf{G}_{t}(x)$}

If $g_{t}(x)$ is the first derivative of $G_{t}(x)$, Laplace transforms of these functions are expressed:

$$
\begin{gathered}
L_{g}(s, t)=\int_{0}^{\infty} e^{-s y} g_{t}(y) d y \\
L_{G}(s, t)=\int_{0}^{\infty} e^{-s y} G_{t}(y) d y=\frac{L_{g}(s, t)}{s}
\end{gathered}
$$

using the properties of the Laplace transform.

Theorem 2. The Laplace Transform of $g_{t}^{I . a}(x)$, d.f. of the random variable $-\ln (V(t))$, is the solution of the following Volterra integral equation of the second kind

$$
\begin{aligned}
L_{g I . a}(s, t) & =\xi(\mathrm{st})(1-W(t))+\int_{0}^{t} \xi(\mathrm{s} \tau) w(\tau) L_{g I . a}(s, t-\tau) d \tau \\
s & >0 \quad t>0
\end{aligned}
$$

where $\xi(\varkappa)$ is the Laplace transform of $f(x)$, d.f. of the instantaneous rates of interest $\delta_{i}$.

Proof. The Laplace transform of the former function could be written

$$
\begin{gathered}
L_{g I . a}(s, t)= \\
\int_{0}^{\infty} e^{-s \delta_{1} t_{1}} f\left(\delta_{1}\right) d \delta_{1} \int_{0}^{\infty} \int_{0}^{\infty} \cdots \int_{0}^{\infty} e^{-s\left[\delta_{2} t_{2}+\cdots+\delta_{n-1} t_{n-1}+\delta_{n}\left(\left(t-t_{1}\right)-\sum_{j=2}^{n-1} t_{j}\right)\right]} \\
f\left(\delta_{2}\right) \cdots f\left(\delta_{n}\right) d \delta_{2} \cdots d \delta_{n}= \\
\int_{0}^{\infty} e^{-s \delta_{1} t_{1}} f\left(\delta_{1}\right) d \delta_{1} L_{g I . a}\left(s, t-t_{1}\right)
\end{gathered}
$$



tained:

if we denote $\xi\left(\right.$ st $\left._{1}\right)=\int_{0}^{\infty} e^{-s \delta_{1} t_{1}} f\left(\delta_{1}\right) d \delta_{1}$, the following expression is ob-

$$
L_{g I . a}(s, t)=\xi\left(\mathrm{st}_{1}\right) L_{g I . a}\left(s, t-t_{1}\right)
$$

which could be considered as the Laplace transform of the d.f. $g_{t}^{I \cdot a}(x)$ conditioned that the first waiting time is $t_{1}$.

A renewal argument will be used now,

a) If $t_{1} \geq t$ then $L_{g I . a}(s, t)=\xi(\mathrm{st})$ with a probability of $1-W(t)$

b) If $t_{1}=\tau<0$ then $L_{g s . a}(s, t)=\xi(\mathrm{s} \tau) L_{g I . a}(s, t-\tau)$ weighted with the density function $w(\tau)$.

leading to the integral equation 3.2

Theorem 3. The Laplace Transform of $g_{t}^{I . b}(x)$, d.f. of the random variable $-\ln (V(t))$, can be expressed

$$
\begin{gathered}
L_{g I . b}(s, t)= \\
\int_{0}^{\infty} e^{-s x} g_{t}^{I . b}(x) d x= \\
\frac{1}{\delta_{1}^{*} W_{1}\left(\frac{t}{\delta_{1}^{*}}\right)} \int_{0}^{\delta_{1}^{*} t} e^{-s \tau} L_{g I . a}\left(s, t-\frac{\tau}{\delta_{1}^{*}}\right) w_{1}\left(\frac{\tau}{\delta_{1}^{*}}\right) d \tau \quad s>0 \quad t>0
\end{gathered}
$$

Proof. It is trivial using the former Theorem and the properties of the Laplace transform

Theorem 4. The Fourier Transform of $g_{t}^{I I}(x)$,d.f. of the random variable $\ln (V(t))$, is the solution of the following Volterra integral equation of the second kind

$$
\begin{aligned}
\mathfrak{F}_{g^{I I}}(s, t)= & e^{I s \delta_{0} t} \zeta(\mathrm{st})(1-W(t)) \\
& +\int_{0}^{t} \zeta(\mathrm{s} \tau) e^{I s \delta_{0} \tau} w(\tau) \mathfrak{F}_{g^{I I}}(s, t-\tau) d \tau \\
s \in & (-\infty, \infty) \quad t>0
\end{aligned}
$$

where $\zeta(\varkappa)$ is the Fourier Transform of $f(x)$, d.f. of the changes in the instantaneous rates of interest $\varepsilon_{i}$.

Proof. Defining the random variable

$$
\rho(t)=\varepsilon_{1} t+\varepsilon_{2}\left(t-t_{1}\right)+\cdots+\varepsilon_{n}\left(t-\sum_{j=1}^{n-1} t_{j}\right)
$$


then

$$
\gamma^{I I}(t)=\delta_{0} t+\rho(t)
$$

and

$$
\begin{gathered}
\dot{\leftrightarrow}_{t}^{I I}(x)=P\left\{\gamma^{I I}(t) \leq x\right\}=P\left\{\gamma^{I I}(t) \leq x\right\}= \\
P\left\{\delta_{0} t+\rho(t) \leq x\right\}=P\left\{\rho(t) \leq\left(x-\delta_{0} t\right)\right\}= \\
=R_{t}\left(x-\delta_{0} t\right) \quad x \in(-\infty,+\infty)
\end{gathered}
$$

the Fourier transform of $G_{t}^{I I}(x)$ can be expressed

$$
\begin{gathered}
\mathfrak{F}_{G^{I I}}(s, t)=\int_{-\infty}^{+\infty} e^{I s y} G_{t}^{I I}(y) d y= \\
\int_{-\infty}^{+\infty} e^{I s y} R_{t}\left(y-\delta_{0} t\right) d y=e^{I s \delta_{0} t} \int_{-\infty}^{+\infty} e^{I s y} R_{t}(y) d y= \\
e^{I s \delta_{0} t} \mathfrak{F}_{R}(s, t)=e^{I s \delta_{0} t} \frac{\mathfrak{F}_{r}(s, t)}{-I s} \quad s \in(-\infty,+\infty) \quad t>0
\end{gathered}
$$

and

$$
\mathfrak{F}_{g^{I I}}(s, t)=e^{I s \delta_{0} t} \mathfrak{F}_{r}(s, t)
$$

where $r_{t}(y)$ is the first derivative of $R_{t}(y)$ and

$$
\begin{gathered}
\mathfrak{F}_{r}(s, t)=\int_{-\infty}^{+\infty} e^{I s y} r_{t}(y) d y \\
\mathfrak{F}_{R}(s, t)=\int_{-\infty}^{+\infty} e^{I s y} R_{t}(y) d y=\frac{\mathfrak{F}_{r}(s, t)}{-I s}
\end{gathered}
$$

We will focus now our attention on $\mathfrak{F}_{r}(s, t)$. It is obvious that

$$
\begin{gathered}
\mathfrak{F}_{r}(s, t)=\int_{-\infty}^{+\infty} \int_{-\infty}^{+\infty} \cdots \int_{-\infty}^{+\infty} e^{I s\left[\varepsilon_{1} t+\varepsilon_{2}\left(t-t_{1}\right)+\cdots+\varepsilon_{n}\left(t-\sum_{j=1}^{n-1} t_{j}\right)\right]} h\left(\varepsilon_{1}\right) \cdots \\
\int_{-\infty}^{+\infty} e^{I s \varepsilon_{1} t} h\left(\varepsilon_{1}\right) d \varepsilon_{1} \int_{-\infty}^{+\infty} \int_{-\infty}^{+\infty} \cdots \int_{-\infty}^{+\infty} e^{I s\left[\varepsilon_{2}\left(t-t_{1}\right)+\varepsilon_{3}\left(\left(t-t_{1}\right)-t_{2}\right)+\cdots+\varepsilon_{n}\left(\left(t-t_{1}\right)-\sum_{j=2}^{n-1} t_{j}\right)\right]} \\
f\left(\varepsilon_{2}\right) \cdots f\left(\varepsilon_{n}\right) d \varepsilon_{2} \cdots d \varepsilon_{n}=
\end{gathered}
$$




$$
\int_{0}^{\infty} e^{I s \varepsilon_{1} t_{1}} f\left(\varepsilon_{1}\right) d \varepsilon_{1} \mathfrak{F}_{r}\left(s, t-t_{1}\right)
$$

using the function $\zeta\left(s t_{1}\right)=\int_{0}^{\infty} e^{I s \varepsilon_{1} t_{1}} f\left(\varepsilon_{1}\right) d \varepsilon_{1}$ we can finally get

$$
\mathfrak{F}_{r}(s, t)=\zeta\left(s t_{1}\right) \mathfrak{F}_{r}\left(s, t-t_{1}\right)
$$

and using the renewal argument that was used in Model I.a

a) If $t_{1} \geq t$ then $\mathfrak{F}_{r}(s, t)=\zeta($ st) with a probability of $1-W(t)$

b) If $t_{1}=\tau<0$ then $\mathfrak{F}_{r}(s, t)=\zeta(\mathrm{s} \tau) \mathfrak{F}_{r}(s, t-\tau)$ weighted with the density function $w(\tau)$

leading in this case to the following renewal equation

$$
\begin{aligned}
\mathfrak{F}_{r}(s, t) & =\zeta(\mathrm{st})(1-W(t))+\int_{0}^{t} \zeta(\mathrm{s} \tau) w(\tau) \mathfrak{F}_{r}(s, t-\tau) d \tau \\
s & \in(-\infty, \infty) \quad t>0
\end{aligned}
$$

similar to the one that we obtained in the case of Laplace transform in the Model I.a 3.2. Multiplying both sides by $e^{I s \delta_{0} t}$ we can write

$$
\begin{aligned}
e^{I s \delta_{0} t} \mathfrak{F}_{r}(s, t)= & e^{I s \delta_{0} t} \zeta(\mathrm{st})(1-W(t)) \\
& +\int_{0}^{t} \zeta(\mathrm{s} \tau) e^{I s \delta_{0} \tau} w(\tau) e^{I s \delta_{0}(t-\tau)} \mathfrak{F}_{r}(s, t-\tau) d \tau \\
s \in & (-\infty, \infty) \quad t>0
\end{aligned}
$$

and finally

$$
\begin{aligned}
\mathfrak{F}_{g^{I I}}(s, t) & =e^{I s \delta_{0} t} \zeta(\mathrm{st})(1-W(t))+\int_{0}^{t} \zeta(\mathrm{s} \tau) e^{I s \delta_{0} \tau} w(\tau) \mathfrak{F}_{g^{I I}}(s, t-\tau) d \tau \\
s & \in(-\infty, \infty) \quad t>0
\end{aligned}
$$

We shall solve the Volterra equations of the second kind of $3.2,3.4$ using Laplace transforms.

Let us define explicitly now the Laplace transform operator:

$$
\mathfrak{L}[k(x), x, z]=\int_{0}^{\infty} e^{-z x} k(x) d x
$$

Theorem 5. The Laplace transform of $g_{t}^{I . a}(x)$,d.f. of the random variable $-\ln (V(t)), L_{g^{I . a}}(s, t)$, can be obtained as the inverse Laplace transform of the function:

for a fixed value of $s$.

$$
\mathcal{L}^{I . a}(s, z)=\frac{\mathfrak{L}[\xi(\mathrm{st})(1-W(t)), t, z]}{1-\mathfrak{L}[\xi(\mathrm{st}) w(t), t, z]} \quad z>0
$$


Proof. Using the Laplace transform operator 3.7 in the integral equation 3.2 over $t$,

$$
\begin{gathered}
\mathfrak{L}\left[L_{g I . a}(s, t), t, z\right]=\mathfrak{L}[\xi(\mathrm{st})(1-W(t)), t, z] \\
+\mathfrak{L}[\xi(\mathrm{st}) w(t), t, z] \mathfrak{L}\left[L_{g I . a}(s, t), t, z\right]
\end{gathered}
$$

finally

$$
\mathfrak{L}\left[L_{g I . a}(s, t), t, z\right]=\frac{\mathfrak{L}[\xi(\mathrm{st})(1-W(t)), t, z]}{1-\mathfrak{L}[\xi(\mathrm{st}) w(t), t, z]}=\mathcal{L}^{I . a}(s, z)
$$

then the Laplace transform using variable $\mathrm{t}$ of $L_{g I . a}(s, t)$ will be $\mathcal{L}^{I . a}(s, z)$. we can use the inverse Laplace transform in order to obtain $L_{g^{I . a}}(s, t)$,

$$
L_{g^{I . a}}(s, t)=\frac{1}{2 \pi I} \int_{c-I \infty}^{c+I \infty} e^{-z t} \mathcal{L}^{I . a}(s, z) d z
$$

where $\mathrm{c}$ is a parameter that exceeds the real part of all singularities of $\mathcal{L}^{I . a}(s, z)$.

Theorem 6. The Fourier transform of $g_{t}^{I I}(x)$, d.f. of the random variable $\ln (V(t)), \mathfrak{F}_{g^{I I}}(s, t)$, can be obtained as the inverse Laplace transform of the function:

$$
\mathcal{L}^{I I}(s, z)=\frac{\mathfrak{L}\left[e^{I s \delta_{0} t} \zeta(\mathrm{st})(1-W(t)), t, z\right]}{1-\mathfrak{L}\left[e^{I s \delta_{0} t} \zeta(\mathrm{st}) w(t), t, z\right]} \quad z>0
$$

for a fixed value of $s$.

Proof. Using again the Laplace transform operator 3.7 in 3.4

$$
\begin{aligned}
& \mathfrak{L}\left[\mathfrak{F}_{g^{I I}}(s, t), t, z\right]=\mathfrak{L}\left[e^{I s \delta_{0} t} \zeta(\mathrm{st})(1-W(t)), t, z\right] \\
& \quad+\mathfrak{L}\left[e^{I s \delta_{0} t} \zeta(\mathrm{st}) w(t), t, z\right] \mathfrak{L}\left[\mathfrak{F}_{g^{I I}}(s, t), t, z\right]
\end{aligned}
$$

leading to

$$
\mathfrak{L}\left[\mathfrak{F}_{g^{I I}}(s, t), t, z\right]=\frac{\mathfrak{L}\left[e^{I s \delta_{0} t} \zeta(\mathrm{st})(1-W(t)), t, z\right]}{1-\mathfrak{L}\left[e^{I s \delta_{0} t} \zeta(\mathrm{st}) w(t), t, z\right]}=\mathcal{L}^{I I}(s, z)
$$

we can use the inverse Laplace transform in order to obtain $\mathfrak{F}_{g^{I I}}(s, t)$

$$
\mathfrak{F}_{g^{I I}}(s, t)=\frac{1}{2 \pi I} \int_{c-I \infty}^{c+I \infty} e^{-z t} \mathcal{L}^{I I}(s, z) d z
$$

where $\mathrm{c}$ is a parameter that exceeds the real part of all singularities of $\mathcal{L}^{I I}(s, z)$ 


\section{MOMENTS OF THE DISTRIBUTIONS}

In Models I.a.and I.b, the moments of the probability distribution of $V(t)$ (d.f. $\mathrm{d}_{t}(\mathrm{x})$ ) can be expressed using 1.2 and 1.4

$$
\begin{gathered}
E[V(t)]^{i}=a_{i}(t)=\int_{0}^{\infty}(y)^{i} d_{t}(y) d y=\int_{0}^{\infty}\left(e^{-x}\right)^{i} g_{t}^{I . a(I . b)}(x) d x= \\
\int_{0}^{\infty} e^{-i x} g_{t}^{I . a(I . b)}(x) d x=L_{g^{I . a(I . b)}}(i, t)
\end{gathered}
$$

and in Model II

$$
\begin{gathered}
E[V(t)]^{i}=a_{i}^{I I}(t)=\int_{-\infty}^{+\infty}(y)^{i} d_{t}(y) d y=\int_{-\infty}^{+\infty}\left(e^{-x}\right)^{i} g_{t}^{I I}(x) d x= \\
\int_{-\infty}^{+\infty} e^{\left(-\frac{i}{I}\right) I x} g_{t}^{I I}(x) d x=\mathfrak{F}_{g^{I I}}\left(-\frac{i}{I}, t\right)
\end{gathered}
$$

The moments can be obtained using analytical solutions or numerical approximations of the inverse Laplace transforms 3.9 and 3.11 and 3.3 in Model I.b.

It is obvious that in most cases, analytical inverse Laplace transforms will be hard to obtain and, consequently, numerical techniques must be used.

\section{DISTRIBUTION FUNCTION OF $\mathrm{V}(\mathrm{t})$}

Let us remember expression 1.4

$$
P\{V(t) \leq x\}=1-G_{t}(-\ln (x))
$$

In Models I.a and I.b the functions $L_{G^{I . a}}(s, t)$ and $L_{G^{I . b}}(s, t)$ represent the Laplace transforms of functions $G_{t}^{I . a}(x)$ and $G_{t}^{I . b}(x)$ respectively. Using expressions 3.2 and 3.3 and 3.1

$$
L_{G^{I . a(I . b)}}(s, t)=\frac{L_{g^{I . a(I . b)}}(s, t)}{s}
$$

where $L_{g^{I . a}}(s, t)$ can be obtained from 3.9 analytical or numerically, and $L_{g^{I . b}}(s, t)$ from 3.3 .

Then inverting Laplace transform again could lead us to analytical expressions or approximations of $G_{t}^{I . a(I . b)}(x)$,

$$
G_{t}^{I . a(I . b)}(x)=\frac{1}{2 \pi I} \int_{d-I \infty}^{d+I \infty} e^{-x s} L_{G^{I . a(I . b)}}(s, t) d s
$$

where $d$ is a number that exceeds the real part of all the singularities of $L_{G^{I, a(I, b)}}(s, t)$. 
In Model II., $\mathfrak{F}_{G^{I I}}(s, t)$ stands for the Fourier transform of $G_{t}^{I I}(x)$. Using $3.10 \mathrm{a}$ and 3.6

$$
\mathfrak{F}_{G^{I I}}(s, t)=\frac{\mathfrak{F}_{g^{I I}}(s, t)}{-I s}
$$

where $\mathfrak{F}_{g^{I I}}(s, t)$ can be obtained from 3.11 analytical or numerically.

Finally, we will use the inverse Fourier transform

$$
G_{t}^{I I}(x)=\frac{1}{2 \pi} \int_{-\infty}^{+\infty} e^{-I s x} \mathfrak{F}_{G^{I I}}(s, t) d s
$$

\section{APPLICATIONS TO TERM INSURANCE}

Let us now apply the results obtained above to a term insurance of $m$ years. The random variable $Z$ represents the present value of a term insurance( see Bowers et al.(1986)) of $m$ years where the actualization factor $V(t)$ follow the considerations made in the introduction,

$$
\begin{array}{cl}
Z=c(K+1) V(K+1) & K=0,1, \cdots, m-1 \\
0 & K=m, \cdots
\end{array}
$$

where $K$ is the curtate future-life of a person of age $\mathrm{x}$ and

$$
P\{K=k\}={ }_{k} p_{x} q_{x+k}
$$

The function $c(K+1)$ models the amount paid at the end of the year of death, also known as benefit function. It is supposed to be deterministic.

We should bear in mind that the actualization factor at time $K+1, V(K+$ 1 ), is now a random variable.

Let us now express the expected value and variance of $\mathrm{Z}$ using the well known formulas

$$
\begin{gathered}
E[Z]=E_{K}\left[E_{V}[Z \mid K]\right] \\
\operatorname{Var}[Z]=E_{K}\left[\operatorname{Var}_{V}[Z \mid K]\right]+\operatorname{Var}_{K}\left[E_{V}[Z \mid K]\right]
\end{gathered}
$$

where

$$
\begin{gathered}
E_{V}[Z \mid K]=E[c(K+1) V(K+1)]=c(K+1) a_{1}(K+1) \\
\operatorname{Var}_{V}[Z \mid K]=\operatorname{Var}[c(K+1) V(K+1)]= \\
(c(K+1))^{2}\left(a_{2}(K+1)-\left(a_{1}(K+1)\right)^{2}\right)
\end{gathered}
$$


that can be obtained from 4.1 and 4.2 .

Finally,

$$
E[Z]=E_{K}\left[c(K+1) a_{1}(K+1)\right]=\sum_{i=0}^{m-1} c(i+1) a_{1}(i+1)_{i} p_{x} q_{x+i}
$$

and after some simple simplifications

$$
\begin{gathered}
\operatorname{Var}[Z]=E_{K}\left[(c(K+1))^{2}\left(a_{2}(K+1)-\left(a_{1}(K+1)\right)^{2}\right)\right]+ \\
+\operatorname{Var}_{K}\left[c(K+1) a_{1}(K+1)\right]= \\
\sum_{i=0}^{m-1}(c(i+1))^{2} a_{2}(i+1){ }_{i} p_{x} q_{x+i}-(E[Z])^{2}
\end{gathered}
$$

Using the approach stated in this paper the distribution function of the probability distribution of $\mathrm{Z}$ can also be obtained. Using the Total probability Theorem

$$
\begin{aligned}
P\{Z \leq y\} & =\sum_{i=0}^{m-1} P\{V(i+1) c(i+1) \leq y\}_{i} p_{x} q_{x+i} \\
+\sum_{i=m}^{\infty}{ }_{i} p_{x} q_{x+i} & =\sum_{i=0}^{m-1} D_{i+1}\left(\frac{y}{c(i+1)}\right){ }_{i} p_{x} q_{x+i}+_{m} p_{x}
\end{aligned}
$$

This last result can be of high interest when the law of large numbers cannot be applied.

\section{CONCLUSIONS}

The approach presented in this work ( see expression 1.1) with random rates of interest $\delta_{i}$ that remain fixed during certain waiting times $t_{i}$, can be considered more suitable to situations when the stochastic structure of $\mathrm{d} \delta(t)$ does not allow continuous changes in $\delta(t)$ ( as it is common in many models used in actuarial literature ).

Using simple tools from spectral methods, Laplace and Fourier transforms and simple renewal equations, with Theorems 2 through 6 , we found elegant expressions for the moments of the probability function of the discount factor $\mathrm{V}(\mathrm{t}), 4.1$ and 4.2 , and of a term insurance, 6.5 and 6.6.

The possibility of obtain the distribution functions of the discount factor and term insurance using 5.2 5.4 6.7 make this approach really appealing, specially when the risk of an insurance should be assessed and the conditions for a normal approximation using the law of large numbers do not exist (e.g.: small amount of policies) 


\section{NUMERICAL ILLUSTRATION}

We will get approximations of $D_{t}(x)$, distribution function of the discount factor $V(t)(t=1, t=5$ and $t=10)$, for several values of $x$ using model I.a.

The distributions of the waiting times will be exponential

$$
w(x)=\lambda e^{-\lambda x} \quad x>0
$$

and $\lambda=1$.

The instantaneous rate of payments $\delta_{i}$ will follow a Gamma distribution

$$
f(y)=\frac{a^{b}}{\Gamma(b)} y^{b-1} e^{-a y} \quad y>0
$$

with $a=1240$ and $b=72$, then the expected value $\mu \simeq \ln (1.06)$ and the standard deviation $\sigma=0.006$ and

$$
P\left\{\delta_{i} \in(\ln (1.04), \ln (1.08))\right\} \simeq 1
$$

this last expression means that the rate of interest will fluctuate inside the interval $(0.04,0.08)$ with a probability very close to 1 .

The values of $D_{t}(x)$ were obtained using the Gaver-Stehfest algorithm twice, first in 3.8a getting $L_{G^{I . a}}(s, t) 5.1$ and later in 5.2 to obtain $G_{t}^{I . b}(x)$ and finally substituting in 1.4

With the assumptions made above the integrals used in the calculations have an explicit formula, see for example Gradshteyn and Ryzhik(1994). The Gaver-Stehfest method is a very efficient tool of inverting Laplace transform under certain conditions of smooth behavior, Davies and Martin(1979). A very simple procedure made in Maple $\mathrm{V}$ was implemented to obtain the figures.

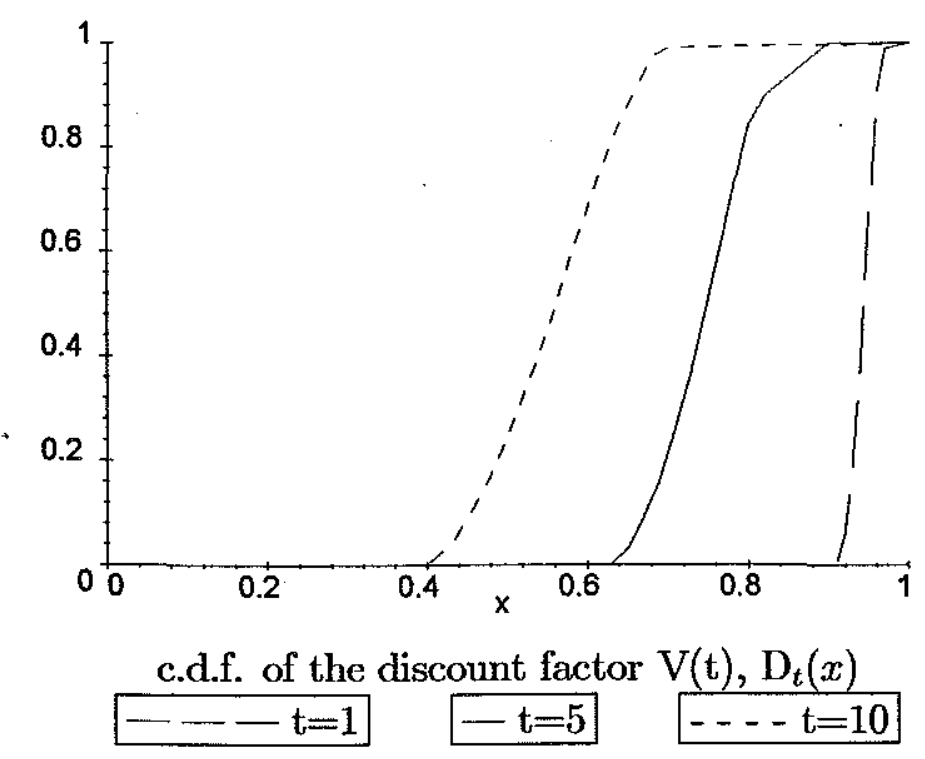




\section{REFERENCES}

[1] Abramowitz, M. and Stegun, I.A. (1972). Handbook of Mathematical Functions. New York, N.Y. Dover Publications.

[2] Ang, A. and Sherris, M. (1997). Interest Rate Management: Developments in interest rate term structure modelling for risk management and valuation of interest-rate-dependent cash flows. North American Actuarial Journal. Volume 1, number 2. April, 1997. pgs 1-26

[3] Bowers, N.L., Gerber, H.U., Hickman, J.C. Jones, D.A. and Nesbitt, C.J. (1986) Actuarial Mathematics. Ithasca, Пll.: Society of Actuaries.

[4] Bühlmann, H. (1995). Life insurance with stochastic interest rates. Financial Risk in Insurance. Ed. G. Ottaviani. Springer-Verlag Heidelberg

[5] Davies, B. and Martin, B.(1979). Numerical inversion of the Laplace transform: a survey and comparison of methods. Journal of computational physics 33, pgs. 1-32

[6] Gradshteyn,I. and Ryzhik, I. (1994). Table of integrals, series and products. Academic Press, Inc. San Diego, Ca.

[7] Hürlimann, W. (1993). Methodes Stochastiques d'evaluation du rendiment. Proc. $3^{\text {rd }}$ AFIR international Colloquium. Roma.

[8] Parker, G. (1997). Stochastic Analysis of the interaction between investment and insurance risks. North American Actuarial Journal: Volume 1, number 2. April, 1997. pgs 1-26 